\title{
Safeguarding choice at the end of life
}

Across the world, in countries with permissive or restrictive existing legislation, debates about Euthanasia and Assisted Suicide (EAS) continue to grip politicians, ethicists, physicians and the wider public.

Early debates about EAS focused on whether it could ever be ethical for a physician to actively cause the death of a patient. However, most contemporary writers, including most of the contributors to this special double issue of the JME appear to accept that such actions could, in some circumstances, be ethical. Current debate is mostly focused instead on which actions are permissible, when they are permissible, and what safeguards are necessary to protect the vulnerable.

There are two separate justifications for EAS. The first of these is based on the autonomy of competent patients, on their right to make important decisions about their own lives. Arguably, a decision about continuing or not continuing your life in the face of severe suffering is the most important decision that you could make. Correspondingly, we have strong autonomy based reasons for permitting that choice. (While some Kantians might claim that a decision to die, and thereby to end one's autonomous agency could not be compatible with autonomy and dignity, Michael Cholbi points out (see page 607) that a sophisticated Kantian position on EAS is neither completely restrictive nor permissive). The second justification for EAS is based on the interests of a patient, and a concern that continued life for some individuals may be so extraordinarily and intensely unpleasant that it would be better for them to die.

Many of the safeguards that have been built into existing or proposed legislation have focused on the autonomy justification for euthanasia, and correspondingly on determining whether a person requesting EAS is competent. (For a discussion of the UK Assisted Dying Bill, proposed by Lord Falconer see page 621). Such laws typically exclude patients with mental illness because of concern that their ability to assess their current and future state, and to weigh up their options is impaired. However, in their feature article, Udo Schuklenk and Suzanne van de Vathorst argue that excluding the option of euthanasia for patients with treatment-resistant depression is unjustified (see page 577).
Some such patients, they argue, are competent to make decisions (they can, for example, consent to participate in research). Furthermore, a desire to die could be rational for someone who experiences severe depression, persisting after multiple different therapies have been tried. Approximately $1 / 3$ of Dutch physicians in Bolt et al's study (see page 592) appear to agree with this, indicating a potential willingness to provide EAS for a patient with a psychiatric illness. Commentators on Schuklenk and van de Vathorst agree that some severely depressed patients could be competent. However, identification of these individuals is extremely challenging. Psychiatrists Matthew Broome and Angharad de Cates suggest a particular challenge for Schuklenk and van de Vathorst's proposal (see page 586). Those depressed patients whose appraisal of their future prospects is not clouded or impaired by their illness may not be severe enough to fit into the category of severe depression. Those who have fluctuating illness, and are intermittently competent, may not meet the criteria for being truly treatment-resistant. One source of disagreement between the feature article authors and commentators is how we should respond to uncertainty. Given the difficulty in determining which patients with depression are truly treatment-resistant, and which treatmentresistant patients with a desire to die are actually competent, Govert den Hartogh (see page 588) and Christopher Cowley (see page 585) argue that policy makers and clinicians should err on the side of caution. Schuklenk and van de Vathorst, however, point to the costs of caution.

Here the second justification for EAS becomes highly relevant. Patients with severe treatment-resistant depression may be in a worse position than patients with a terminal illness and severe physical suffering. If EAS is thought to ever be permissible, most people seem to assume that patients who are soon to die and in physical pain have the strongest claim to be given assistance. However, patients with severe depression, by definition, are intensely unhappy. Their illness robs them of the capacity to enjoy most or all of the things that they formerly enjoyed (while patients with a terminal illness may still in many circumstances enjoy the company of family and friends and a range of activities). Where they have had repeated trials of different therapies without reprieve, such patients have no apparent prospect of improvement or a natural end to their suffering. For these patients, the fact that in the absence of EAS they may survive for some time, arguably gives us a stronger interests-based reason to provide them with the option of ending their life.

One argument that Schuklenk and van de Vathorst could have drawn on relates to the relationship between palliation and EAS. Good quality palliative care can provide relief of physical (and mental) suffering in patients with a terminal illness. Indeed, potentially it is always possible to relieve physical suffering with large enough doses of analgesia/sedation. The only question is whether it is possible to provide strong enough analgesia to relieve pain, while still allowing someone a sufficient degree of consciousness to communicate, appreciate their surroundings and enjoy their remaining time. Where that is not possible, there is the option of sedation to unconsciousness until the patient dies (terminal sedation). If terminal sedation is available, the argument of some palliative care professionals that EAS is not necessary to relieve suffering seems to have traction. ${ }^{i}$ However, that argument will not work for patients with severe chronic depression. Palliative care is not typically available for patients with severe mental illness (in the absence of a life-limiting physical illness). Moreover, the patients that Schuklenk and van de Vathorst focus on have already tried all available means to relieve their mental anguish (for example different pharmacotherapies, counseling, electro-convulsive therapy). It seems unlikely that either psychiatrists or palliative care physicians will be willing to offer terminal sedation to severely depressed patients.

${ }^{\mathrm{i}}$ This argument rebuts the interests-based, but not the autonomy based arguments for EAS. Some patients may prefer to choose the manner as well as the timing of their death. They may prefer to end their life in the setting of a terminal illness before palliative care physicians are prepared to offer terminal sedation. Further, they may prefer to die quickly (from EAS) rather than linger in a state of unconsciousness for days or weeks prior to death (with terminal sedation). 
Other papers in this issue also address the relationship between palliative care and EAS. Some have argued that even if EAS is justified in some situations, it should not be permitted in settings where there is inadequate or absent palliative care. Barutta and Vollmann (see page 652) make the plausible case that this risks worsening the state of some. These individuals are suffering from a terminal illness, suffering more because they lack palliative care, and are denied access to the means of relieving their suffering. Lars Materstvedt, a palliative care specialist (see page 655), acknowledges the mixed views of palliative care professionals, even while official organisations such as the European Association of Palliative Care maintain an opposition to EAS. Materstvedt notes that wishes of patients for assistance with dying are often "ambivalent, fluctuating and hypothetical", a point also echoed in den Hartogh's commentary. One argument addressed by Barutta and Vollman, is what they call the 'argument of antagonism' between EAS and palliative care. Specifically, that allowing EAS hinders the development of PC. Chambaere and Bernheim provide some useful empirical evidence against the argument of antagonism (see page 657). Their paper appears to indicate the opposite phenomenon: Belgium, the Netherlands and Luxemburg have increased spending and availability of palliative care compared with neighbouring countries in the last decade.

For both supporters and opponents of EAS, good palliative care is crucial. One important question is how to ensure consistent, appropriate care for dying patients. The UK has, arguably, one of the strongest palliative care services in Europe. ${ }^{\text {ii }}$ Several papers in this issue conduct a post-mortem examination of the Liverpool Care Pathway - a structured guideline for end of life care that was developed by palliative care specialists in Liverpool, and that subsequently attracted considerable negative publicity, a formal inquiry (the Neuberger Review) and was then phased out in the UK.

Anthony Wrigley notes that the review identified problems with the application of the LCP rather than the substance of the pathway (see page 639). Wrigley suggests that the conclusion of the Neuberger review is analogous to someone finding that insulin is sometimes used incorrectly in a harmful way, and concluding that therefore it should be rejected as a therapy. Palliative Care physician David

iiThis does not stop some patients from travelling overseas to access EAS. See the report by Gauthier (see page 611) and accompanying commentaries by Foster (see page 620) and Luley (see page 618).
Mackintosh agrees (see page 650). He makes the plausible claim that health care professionals who were unable to provide appropriate end of life care in the presence of the LCP are unlikely to be able to do so in its absence. However, Mohamad Rady and Joseph Verheijde (see page 644). worry, in particular, about the apparent endorsement of withdrawal of artificial nutrition and hydration as part of the LCP. They feel that this represented an attempt to provide covert active euthanasia. Anna Nowarska, in contrast, argues that withdrawal of artificial nutrition and hydration is different from active euthanasia, and consistent with Catholic teaching (see page 645).

As the papers in this issue highlight, there are major differences in approach to end of life care internationally. This represents a considerable challenge (how do we justify these different approaches, how do we deal with cross-border movement), but also a tremendous opportunity. Our era represents a vast global experiment in care of the dying. There has never been a time with such a range of different approaches and with such intense scrutiny, research and debate. There will doubtless be mistakes made, and those are just as important to note as the successes. Wherever we are, whatever our preferred philosophy, we should take the opportunity to look over the fence at our neighbours and learn. 